\title{
A SMALL CLUSTER APPROACH FOR THE ELECTRONIC DENSITY OF STATES IN AMORPHOUS GERMANIUM
}

\author{
M. A. GRADO-CAFFARO and M. GRADO-CAFFARO \\ C/Julio Palacios 11, 9B 28029-Madrid, Spain
}

(Received 3 July 1997; In final form 22 July 1997)

\begin{abstract}
The electronic density of states for very small clusters of amorphous germanium is calculated by using bonding orbitals. In this context, the Born model is considered as well as Pauling-type hybridized $\mathrm{sp}^{3}$ orbitals.
\end{abstract}

Keywords: Electronic density of states; amorphous germanium; bonding orbitals; Born model

\section{INTRODUCTION}

Due in part to a somewhat scant success of GaAs for microwave applications, Ge becomes again a material to be considered for these applications. In particular, this semiconductor in amorphous state presents interesting features which, in spite of the very large number of papers published on this subject, have not been studied adequately due mainly to the proliferation of obscure procedures in the context of theoretical research. Consequently, research efforts are required in order to solve open problems related to density of states, spectra, etc.

The aim of this paper deals with electronic density of states for amorphous $\mathrm{Ge}$ in the context of quantum-mechanical calculations involving localized pseudo-potential theory [1]. Bonding orbitals and Pauling-type hybridized orbitals are relevant in this theory. Furthermore, the Born model for atomic interaction [1] [2] will be used in the 
following by considering an appropriate relationship between the involved two force constants [3].

\section{THEORY}

Consider two isolated atoms labeled 1 and 2; we formulate the timeindependent Schrödinger equation corresponding to the above system namely:

$$
\hat{H}\left|\phi_{12}\right\rangle=E\left|\phi_{12}\right\rangle
$$

where $\hat{H}_{12}$ stands for hamiltonian, $E$ is energy and $\left|\phi_{12}\right\rangle$ local bonding orbital. A given electron in this orbital is influenced by the potential of perturbation of the atoms 1 and 2; let us denote this potential by $\hat{V}_{12}$. We have:

$$
\hat{H}=\hat{H}_{12}+\hat{V}_{12}
$$

$\hat{H}$ being the total single-electron hamiltonian.

On the other hand, we can write (see Ref. [1], Eq. (3)):

$$
\hat{V}_{12}=\frac{1}{2}(\alpha-\beta)\left|\overrightarrow{u_{1}}-\overrightarrow{u_{2}}\right|^{2}
$$

where $\alpha$ and $\beta$ are force constants; $\overrightarrow{u_{1}}$ and $\overrightarrow{u_{2}}$ are the displacement vectors of the atoms 1 and 2 , respectively. Now we take, for amorphous $\mathrm{Ge},(\alpha / \beta)=(5 / 3)$ (see Ref. [3]) so that formula (3) becomes:

$$
\hat{V}_{12}=\frac{1}{3} \beta\left|\overrightarrow{u_{1}}-\overrightarrow{u_{2}}\right|^{2}
$$

In addition, we have [1] :

$$
\left|\phi_{12}\right\rangle=c\left(\left|\phi_{1 \rightarrow 2}\right\rangle+\left|\phi_{2 \rightarrow 1}\right\rangle\right)
$$

where $c$ is a constant, $\left|\phi_{1 \rightarrow 2}\right\rangle$ is the orbital on atom 1 directed towards atom 2 , and $\left|\psi_{2 \rightarrow 1}\right\rangle$ is the orbital on atom 2 directed towards atom 1; Eq. (5) represents the normalized sum of a Pauling-type hybridized $\mathrm{sp}^{3}$ orbital on each atom.

Considering very small clusters, total electronic density of states can be expressed as follows (see Ref. [1], Eq. (10)):

$$
g(E) \approx \Omega \sum_{n}\left[\left|\int_{R} \psi_{n}(\vec{r}) \phi_{12}(\vec{r}) d^{3} \vec{r}\right|^{2} \delta\left(E-E_{n}\right)\right]
$$


where $\Omega$ is the volume of the cluster; the energy eigenvalues $E_{n}$ and the eigenfunctions $\psi_{n}$ satisfy:

$$
\hat{H}\left|\psi_{n}\right\rangle=E_{n}\left|\psi_{n}\right\rangle
$$

By combining Eq. 5 and Eq. 6, we get:

$$
g(E) \approx c^{2} \Omega \sum_{n}\left[\left|\left\langle\psi_{n}^{*} \mid \phi_{1 \rightarrow 2}\right\rangle+\left\langle\psi_{n}^{*} \mid \phi_{2 \rightarrow 1}\right\rangle\right|^{2} \delta\left(E-E_{n}\right)\right]
$$

with

$$
\left\langle\psi_{n}^{*} \mid \phi_{1 \rightarrow 2}\right\rangle=\int_{R} \psi_{n}(\vec{r}) \phi_{1 \rightarrow 2}(\vec{r}) d^{3} \vec{r}
$$

and

$$
\left\langle\psi_{n}^{*} \mid \phi_{2 \rightarrow 1}\right\rangle=\int_{R} \psi_{n}(\vec{r}) \phi_{2 \rightarrow 1}(\vec{r}) d^{3} \vec{r}
$$

Using numerical values for a 5-atom cluster of amorphous $\mathrm{Ge}$ and taking into account Eqs. (1), (2), (3), (4) and (8), after various calculations, we have obtained the plot of Figure 1.

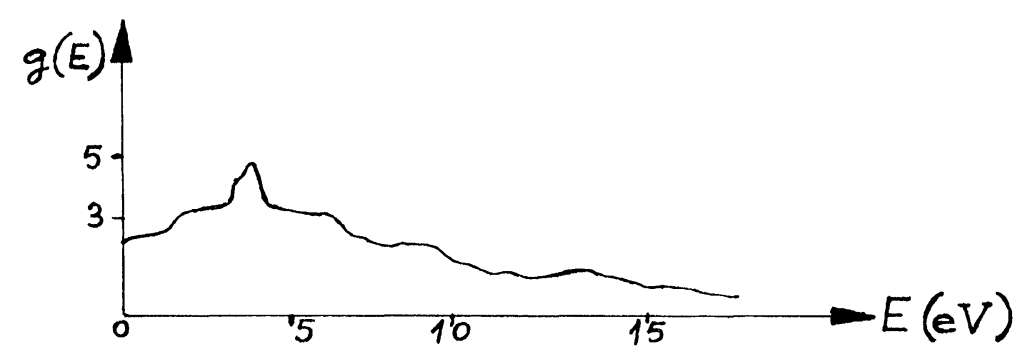

FIGURE 1 Total electronic density of states against energy for a 5-atom cluster of a-Ge. 
This plot presents a certain similarity to Figure 2 of Ref. [4]; this figure refers to a 47-atom cluster of a-Ge. For more details on this type of cluster, see Refs. [5, 6].

\section{CONCLUDING REMARKS}

We have obtained $g(E)$ as a curve with a significant peak at $E \approx$ $3.73 \mathrm{eV}$; at this value, we have found $g \approx 4.92$ (arbitrary units). On the other hand, a relevant fact is remarkable: our previous theoretical formulation is clearly different from other approaches based upon somewhat weak assumptions related to empirical techniques that become unuseful for solving the major problems associated with atom clusters.

\section{References}

[1] Grado-Caffaro, M. A. and Grado-Caffaro, M. (1994). Act. Pass. Electronic Comp., 16, $105-107$.

[2] Thorpe, M. F. (1974). AIP Conf. Proc. No. 20, 267-273.

[3] Alben, R. (1974). AIP Conf. Proc. No. 20, 255.

[4] Tong, B. Y. (1974). AIP Conf. Proc. No. 20, 147.

[5] Henderson, D. and Herman, F. (1972). J. Non-Cryst. Solids, 8-10, 359.

[6] Grado-Caffaro, M. A. and Grado-Caffaro, M. (unpublished). 

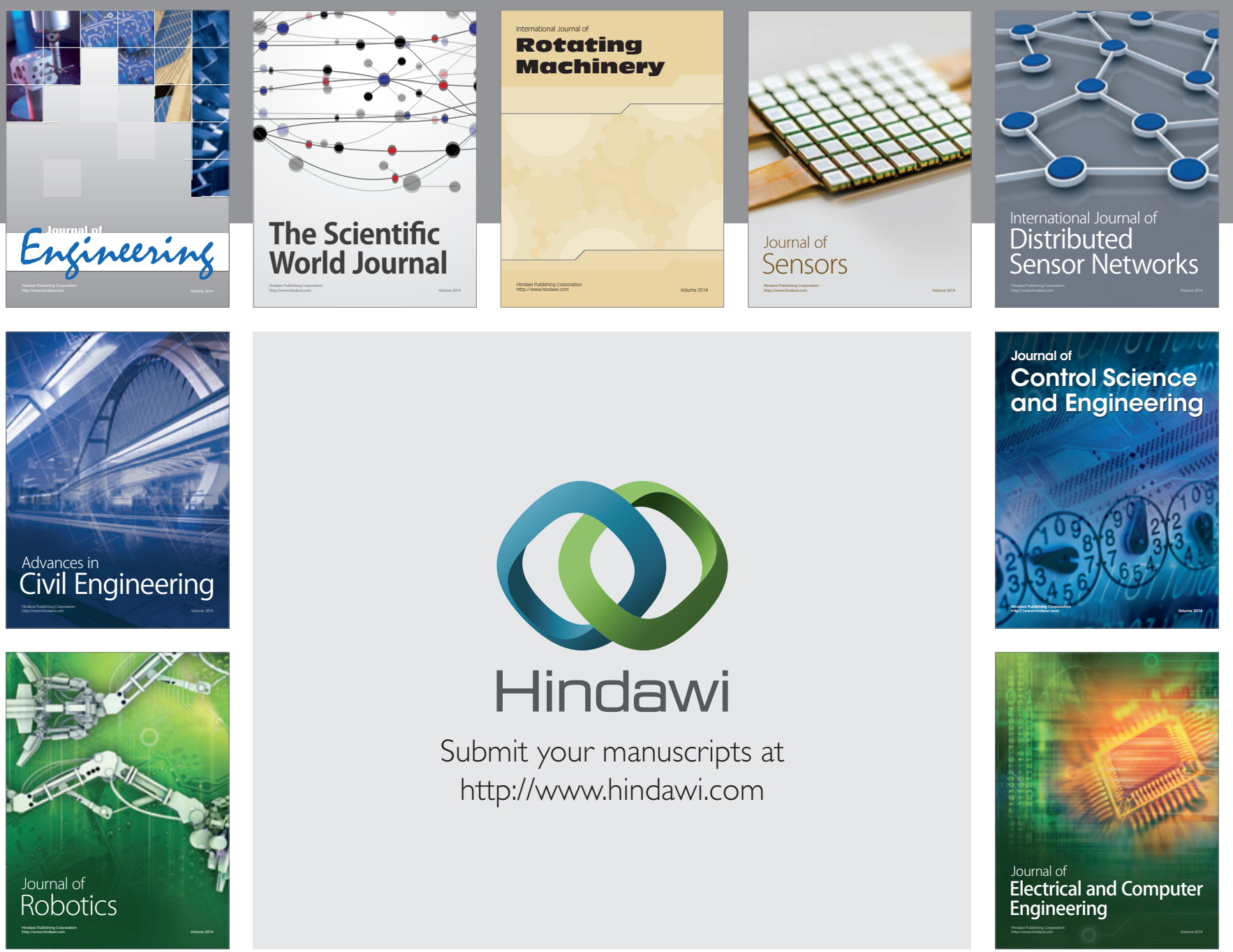

Submit your manuscripts at

http://www.hindawi.com
